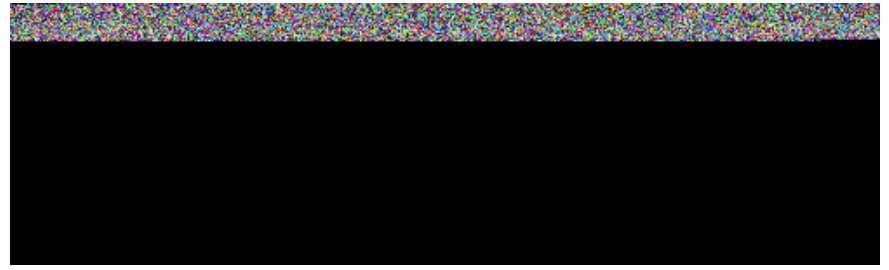

This information is current as of April 26, 2023.

\title{
Pacemakers in MRI for the Neuroradiologist: Revisited
}

\section{E. Kanal}

AJNR Am J Neuroradiol 2018, 39 (5) E54-E55

doi: https://doi.org/10.3174/ajnr.A5565

http://www.ajnr.org/content/39/5/E54 


\section{Pacemakers in MRI for the Neuroradiologist: Revisited}

I read with interest the excellent article by Korutz et al ${ }^{1}$ regarding their approach to MR imaging of pacemakers. The authors are to be praised for their logical and stepwise approach to device safety in the MR imaging environment. I assume that the timing of the publication of this article likely explains the omission of a reference to the sentinel publication on this topic. ${ }^{2}$ That comprehensive publication also provides almost unprecedented strength of recommendations and quality of evidence quantifications.

I would like to suggest other approaches to several opinions promulgated by Korutz et al.

The 6-week waiting period of the MR imaging safety labeling condition historically results predominantly because during Phase III preclinical trials, device manufacturers did not want acute/subacute surgical postimplantation adverse events (such as spontaneous lead dislodgment) to be confused with MR imagingrelated adverse events. Because these were the conditions under which these devices were studied, this same "6-week postimplantation" wording finds its way into the conditions of FDA approval. While scanning before 6 weeks postimplantation would indeed be off-label, it would be a shame for a clinically indicated or required MR imaging examination to be canceled simply because the study was needed earlier than 6 weeks postimplantation.

The article is directed to neuroradiologists, and it is indeed likely that the typical head or spine study they perform would expose at least part of the cardiovascular implantable electronic device (CIED) and/or its leads to clinically relevant transmitted radiofrequency $(\mathrm{RF})$ and/or MR imaging gradient (ie, $\mathrm{dB} / \mathrm{dt}$ ) energies. Yet a clinically indicated MR imaging study might well be safely performed on a patient with a CIED if the requested MR imaging examination were 1 ) in a region where the anticipated gradient $\mathrm{dB} / \mathrm{dt}$ exposure to the CIED and its leads would be minimal; and 2) there would be no significant exposure of the CIED or its components to transmitted RF energies or significant induced electric field pathways. These conditions might exist for imaging of the leg, knee, ankle, foot, and so forth of a typical-height adult, though such regions would admittedly not be routinely examined by a neuroradiologist.

The relatively recent FDA approval of the MR imaging condi-

http://dx.doi.org/10.3174/ajnr.A5565 tionally safe labeling for several brands of leadless intracardiac pacing devices is also noteworthy because such leadless devices negate most of the MR safety concerns raised in the article.

Induced Lenz forces from rapid motion of CIEDs through static magnetic spatial gradients (even for nonferrous electrically conductive materials) suggest that it might be appropriate to recommend that all patients and health care workers with implants move slowly within zone IV, especially when near or in the MR imaging scanner itself.

The authors note significant hesitation "for patients who are not awake and alert for the MR imaging examination, such as those who ... are unable to report pain or discomfort during the examination ...." Because the primary CIED-related potential acute concerns associated with the gradient and RF fields are arrhythmogenesis and endocardial thermogenic damage/edema at the endocardial contact points of the intracardiac leads, it is unclear that a conscious responsive patient would be able to detect, let alone timely report, pain or discomfort in case of either thermal endocardial damage or arrhythmogenesis.

The authors conclude, "A few absolute contraindications remain for performing MR imaging in a patient with a CIED” (eg, "a device that was implanted $<6$ weeks before the MR imaging examination"). Our responsibility as physicians is to perform a benefit-risk assessment for each patient's clinical scenario before providing blanket approvals_ or cancellations_of any examination. MR imaging of patients with CIEDs is no exception. With appropriate clinical supervision from our electrophysiology colleagues and our ability to markedly decrease transmitted RF energies and today even imaging gradient $\mathrm{dB} / \mathrm{dt}$ as needed, one might reasonably accept for MR imaging a patient with an unlabeled device with suspected cord compression, epidural abscess, device implanted for $<6$ weeks, and so forth. Alternatively, for even elective studies, the requested examination may be in an anatomic location for which no significant RF or imaging gradient exposure to the CIED will occur, regardless of the CIED label or the presence/absence of lead breaks. Therefore, as long as we are able to assess potential benefits and risks to our patients, I respectfully submit that absolute contraindications and carte blanche rejection of patients with devices from clinically indicated MR imaging studies without some level of case review and relative benefit-risk 
ratio assessment should no longer be recommended or implemented.

Disclosures: Emanuel Kanal—UNRELATED: Consultancy: Medtronic, St. Jude Medical.

\section{REFERENCES}

1. Korutz AW, Obajuluwa A, Lester MS, et al. Pacemakers in MRI for the neuroradiologist. AJNR Am J Neuroradiol 2017;38:2222-30 CrossRef Medline
2. Indik JH, Gimbel JR, Alkmim-Teixeira R, et al. 2017 HRS expert consensus statement on magnetic resonance imaging and radiation exposure in patients with cardiovascular implantable electronic devices. Heart Rhythm 2017;14:e97-e153 CrossRef Medline

(D)E. Kanal

Magnetic Resonance Services Department of Radiology University of Pittsburgh Medical Center University of Pittsburgh Pittsburgh, Pennsylvania 